\title{
Phase I study of nanoparticle albumin-bound paclitaxel, carboplatin and trastuzumab in women with human epidermal growth factor receptor 2-overexpressing breast cancer
}

\author{
KENJI TEZUKA $^{1,14}$, TSUTOMU TAKASHIMA ${ }^{2}$, SHINICHIRO KASHIWAGI ${ }^{2}$, HIDEMI KAWAJIRI ${ }^{3}$, \\ SHINYA TOKUNAGA ${ }^{4}$, SEIKA TEI ${ }^{5}$, SHIGEHIKO NISHIMURA ${ }^{6}$, SHIGEHITO YAMAGATA $^{6}$, SATORU NODA $^{2}$, \\ TAKEO NISHIMORI ${ }^{7}$, YOKO MIZUYAMA ${ }^{8}$, TAKESHI SUNAMI ${ }^{9}$, KATSUMI IKEDA $^{10}$, \\ YOSHINARI OGAWA $^{10}$, NAOYOSHI ONODA ${ }^{2}$, TETSURO ISHIKAWA ${ }^{11}$, \\ SHINZOH KUDOH ${ }^{12}$, MINORU TAKADA ${ }^{13}$ and KOSEI HIRAKAWA ${ }^{2}$
}

${ }^{1}$ Department of Breast Surgery, National Hospital Organization Kinki-Chuo Chest Medical Center, Sakai, Osaka 591-8555;

${ }^{2}$ Department of Surgical Oncology, Osaka University Graduate School of Medicine, Osaka 545-8585; ${ }^{3}$ Department of Head, Neck and Breast Surgery, Ishikiriseiki Hospital, Higashiosaka, Osaka 579-8026; ${ }^{4}$ Department of Clinical Oncology, Osaka General Hospital, Osaka 534-0021; ${ }^{5}$ Department of Surgery, Seichokai Fuchu Hospital, Izumi, Osaka 594-0076; ${ }^{6}$ Department of Surgery, Sumitomo Hospital, Osaka 530-0005; ${ }^{7}$ Department of Surgery, Ikuwakai Memorial Hospital, Osaka 544-0004; ${ }^{8}$ Department of Surgery, Ohno Memorial Hospital, Osaka 550-0015; ${ }^{9}$ Department of Surgery, Izumi Municipal Hospital, Izumi, Osaka 594-0071; ${ }^{10}$ Department of Breast Surgery, Osaka General Hospital, Osaka 534-0021; ${ }^{11}$ Department of Surgery, Kashiwara Municipal Hospital, Kashiwara, Osaka 582-0005;

${ }^{12}$ Department of Internal Medicine, Osaka Socio-Medical Center Hospital, Osaka 557-0004;

${ }^{13}$ Hanwa Daini Senboku Hospital, Sakai, Osaka 599-8271, Japan

Received October 14, 2016; Accepted January 12, 2017

DOI: $10.3892 / \mathrm{mco} .2017 .1176$

\begin{abstract}
Although the concurrent use of anthracycline-containing chemotherapy and taxane with trastuzumab are considered the treatment of choice for the primary systemic therapy of human epidermal growth factor receptor 2 (HER2)-overexpressing early breast cancer, non-anthracycline regimens, such as concurrent administration of docetaxel and carboplatin with trastuzumab, exhibited
\end{abstract}

Correspondence to: Dr Kenji Tezuka, ${ }^{14}$ Present address: Department of Breast Surgery, Kishiwada City Hospital, 1001 Gakuhara-cho, Kishiwada, Osaka 596-8501, Japan

E-mail: tezuken@gmail.com

Abbreviations: AUC, area under the curve; ECOG, Eastern Cooperative Oncology Group; DLT, dose-limiting toxicity; ER, estrogen receptor; FISH, fluorescence in situ hybridization; HER2, human epidermal growth factor receptor 2; IHC, immunochemistry; MBC, metastatic breast cancer; MTD, maximum tolerated dose; nab-paclitaxel, nanoparticle albumin-bound paclitaxel; PgR, progesterone receptor; PS, performance status; RD, recommended dose; UMIN, University Hospital Medical Information Network

Key words: phase I study, nab-paclitaxel, carboplatin and trastuzumab, breast cancer, stage IIIB or IIIC similar efficacies in a previous study. In addition, tri-weekly treatment with nanoparticle albumin-bound paclitaxel (nab-paclitaxel) resulted in significantly higher response rates and a favorable safety profile compared with standard paclitaxel for metastatic breast cancer patients in another phase III study. Based on these results, a phase I study of combination therapy with nab-paclitaxel, carboplatin and trastuzumab was planned, in order to estimate its efficacy and safety for HER2-overexpressing locally advanced breast cancer. The present study was designed to determine the dose-limiting toxicity (DLT), maximum tolerated dose and recommended dose of this combination treatment in women with HER2-overexpressing locally advanced breast cancer. The starting dose of nab-paclitaxel was $220 \mathrm{mg} / \mathrm{m}^{2}$ (level 1), and the dose was escalated to $260 \mathrm{mg} / \mathrm{m}^{2}$ (level 2). Nab-paclitaxel was administered with carboplatin (area under the curve, $6 \mathrm{mg} / \mathrm{ml} / \mathrm{min}$ ) and trastuzumab tri-weekly. A total of 6 patients were enrolled. Although no DLT was observed during the first cycle, 4 patients developed grade 4 thrombocytopenia, 2 had grade 4 neutropenia and 3 exhibited a grade 4 decrease in hemoglobin levels. In the present phase I study, although no patients experienced DLTs, this regimen was associated with severe hematological toxicities and it was not well tolerated. However, considering the high efficacy and lower risk of cardiotoxicity and secondary carcinogenesis with taxane, platinum and trastuzumab combination therapy, further evaluation of another regimen including weekly administration or a more accurate dose setting should be conducted. 


\section{Introduction}

Locally advanced breast cancer is defined as bulky T3 and T4 tumors of the breast, or breast cancer associated with matted axillary (N2) or supraclavicular (N3) adenopathy. The overall outcome and local control rates have improved markedly with multimodal therapy, including the use of surgery after neoadjuvant chemotherapy and locoregional irradiation. Additional postoperative systemic treatments are determined by primary tumor molecular markers. Breast conservation therapy may be offered to selected patients after downstaging by neoadjuvant chemotherapy. Treatment with induction chemotherapy may improve the prognosis based on pathological response (1).

Although sequential use of anthracycline-containing chemotherapy and taxane with trastuzumab may be considered as the treatment of choice for the primary systemic therapy of human epidermal growth factor receptor 2 (HER2)-overexpressing early breast cancer, non-anthracycline regimens, such as concurrent administration of docetaxel and carboplatin with trastuzumab (TCH regimen), exhibited similar efficacies in a previous study (2). Furthermore, the addition of carboplatin to paclitaxel and trastuzumab improved progression-free survival in HER2-overexpressing metastatic breast cancer (MBC) patients in a randomized phase III study (3). Tri-weekly treatment with nanoparticle albumin-bound paclitaxel (nab-paclitaxel) resulted in significantly higher response rates and a favorable safety profile compared with standard paclitaxel for MBC patients in another phase III study (4). In addition, tri-weekly nab-paclitaxel as first-line therapy for MBC achieved similar response rates compared with tri-weekly docetaxel $\left(100 \mathrm{mg} / \mathrm{m}^{2}\right)$ in the phase II study (5).

Based on these results, a phase I/II study of the combination therapy with nab-paclitaxel, carboplatin and trastuzumab was planned to estimate this regimen's efficacy and safety for HER2-overexpressing locally advanced breast cancer. We herein report the results of phase I of the study.

\section{Patients and methods}

Study design. This was a phase I, open-label, dose-finding multicenter trial conducted at eight sites in Japan. The study protocol and all amendments were approved by the local ethics committees or the Institutional Review Board at each study site. This trial was conducted in accordance with the Japanese Guidelines for Clinical Research of the Ministry of Health, Labor, and Welfare, and the Declaration of Helsinki, as well as other applicable regulatory requirements. All the participants provided written informed consent prior to study entry. The present trial has been registered with the University Hospital Medical Information Network (UMIN) Center (ID: UMIN000007600). This was an investigator-initiated clinical trial that was not supported by any industry funding, nor requested by any organization.

Eligibility criteria. In general, non-treated female patients with primary breast cancer who satisfied the following inclusion criteria were selected for enrollment in the study: i) Histologically confirmed invasive ductal carcinoma, regardless of the hormonal status, ii) stage IIIB or IIIC disease, iii) patient age 20-70 years, iv) patients with at least one measurable lesion, v) cancer tissue specimens were evaluated for HER2 overexpression by immunohistochemistry (IHC; Hercep Test ${ }^{\oplus}$; Dako, Carpinteria, CA, USA) with an IHC score of $3+$ or demonstrated fluorescence in situ hybridization (FISH) positive (>2.2) for HER2 gene amplification, and patients with tumors testing $2+$ by IHC were required to have a positive FISH test, vi) patients with a normal left ventricular ejection fraction ( $\geq 55 \%$ measured by echocardiography or multiple-gated acquisition scan), vii) no abnormal findings on electrocardiography, viii) Eastern Cooperative Oncology Group (ECOG) performance status (PS) of 0-1, ix) absence of severe dysfunction of major organs (white blood cell count $\geq 3,000 / \mathrm{mm}^{3}$; neutrophil count $\geq 2,000 / \mathrm{mm}^{3}$; platelet count $\geq 100,000 / \mathrm{mm}^{3}$; hemoglobin level $\geq 9.0 \mathrm{~g} / \mathrm{dl}$; aspartate aminotransferase $\leq 100 \mathrm{IU} / \mathrm{l}$; alanine aminotransferase $\leq 100 \mathrm{IU} / \mathrm{l}$; total bilirubin $\leq 1.5 \mathrm{mg} / \mathrm{dl}$; serum creatinine $\leq 1.5 \mathrm{mg} / \mathrm{dl}$; serum albumin $\geq 3.0 \mathrm{mg} / \mathrm{dl}$ ), and $\mathrm{x}$ ) all patients provided written informed consent. The exclusion criteria included the following: i) History of severe drug hypersensitivity, ii) inflammatory breast cancer (T4d), iii) active double cancer, iv) infection, diarrhea, ileus, poorly controlled diabetes and severe complications, v) uncontrollable pleural effusion or ascites, vi) peripheral neuropathy, vii) gastrointestinal ulcer or bleeding, viii) severe psychiatric disorder, ix) pregnancy or the possibility of pregnancy, $\mathrm{x}$ ) hepatitis B surface antigen positivity or patients who required nucleoside analogue treatment, or xi) any other reason for which the investigator deemed the patient to be unsuitable for inclusion in this study.

Treatment. The starting dose of nab-paclitaxel was $220 \mathrm{mg} / \mathrm{m}^{2}$ (level 1); the dose was escalated to $260 \mathrm{mg} / \mathrm{m}^{2}$ (level 2). Nab-paclitaxel was administered with 6 area under the curve (AUC) of carboplatin and trastuzumab. Trastuzumab was initially administered at a dose of $8 \mathrm{mg} / \mathrm{kg}$ of body weight, followed by $6 \mathrm{mg} / \mathrm{kg}$. This treatment cycle was repeated every 3 weeks from four to six cycles, for as long as the treating physicians considered the disease to be stable or unless a severe adverse event was observed.

Dose-escalation scheme. Initially, 3 registered patients were assigned to level 1 . If a dose-limiting toxicity (DLT) manifested in at least 1 of these patients during the first cycle, 3 more patients were added to the same level. If $\geq 2$ of these 6 patients developed a DLT, that level was defined as the maximum tolerated dose (MTD). Similarly, if 2 of the initial 3 patients developed a DLT, that level was defined as the MTD.

DLT is defined as the manifestation of any of the following: i) Grade 4 thrombocytopenia, ii) grade 3 thrombocytopenia requiring transfusion, iii) grade 4 neutropenia continuing for $>7$ days, iv) grade 3 neutropenia with fever $\geq 38.5^{\circ} \mathrm{C}$, and v) grade 3 or 4 non-hematological toxicity, excluding nausea, vomiting and diarrhea.

Study assessments. Information on patient baseline characteristics was collected within 28 days prior to the initiation of administration. Clinical response assessment was based on the change in the tumor's longest diameter, as estimated by ultrasonography or computed tomography. Tumor size was measured by imaging studies prior to the first chemotherapy treatment, after the fourth course (mid-treatment) and after the 
last course (final). All adverse events were graded according to the Common Terminology Criteria for Adverse Events, version 4.0 (6). Details of all adverse events and laboratory abnormalities for the treatment were to be recorded on the Case Report Form.

The mean values with $95 \%$ confidence interval (CIs) for progression-free survival were estimated using the Kaplan-Meier method.

\section{Results}

Patient characteristics. Between April, 2012 and September, 2015, 6 patients were enrolled in this study. The patient characteristics are summarized in Table I. The median age was 53 years (range, $41-61$ years) and the majority of patients had an ECOG PS of 0 . Of the 6 patients, 3 were premenopausal and 3 were postmenopausal. In addition, 3 patients received dose level 1 and 3 received dose level 2. The patients received a mean of 4 cycles (range, 3-6 cycles).

MTD determination. No DLTs were observed during the first cycle of treatment for any patient. Therefore, the MTD was not determined.

Toxicity. All the adverse events occurring in $>1$ patient are shown in Table II. Although no DLTs were observed during the first cycle, grade 4 neutropenia occurred in 1 patient at level 1 and 1 patient at level 2 after the second cycle. Similarly, a grade 4 decrease in hemoglobin level occurred in 3 patients at level 1 , and grade 4 thrombocytopenia in 2 patients at level 1. No febrile neutropenia was experienced. Transfusions were performed in all the patients at level 1 and 1 patient at leve 2. Grade 3 muscle pain and arthralgia occurred in the same patient at level 2 during the second cycle.

Efficacy. Tumor shrinkage was observed in all 6 patients and the overall response rate was $100 \%$. The median reduction rate was $61.9 \%$ (range, 39.0-69.9\%). One patient at level 2 exhibited a pathological complete response, including dissected axillary lymph nodes in a postoperative specimen. No metastasis to the axillary lymph nodes was pathologically detected in 4 of the 6 patients.

Although no DLTs were observed in the first cycle of this combination treatment in this phase I study, there was difficulty with safety with repeated administration; therefore, we did not move on to a phase II study.

Clinical outcomes. Lung and supraclavicular lymph node recurrence developed in 2 of the 3 patients at level 1 and in 1 patient at level 2. The mean disease-free survival at all levels was 29 months (range, 8-42 months; 95\% CI: 17-40 months). As all 6 patients remain alive, the median overall survival was not calculated.

\section{Discussion}

To the best of our knowledge, this multicenter phase I trial is the first study to evaluate the addition of nab-paclitaxel to carboplatin and trastuzumab as a primary systemic therapy for women with HER2-overexpressing locally advanced breast cancer.
Table I. Patient characteristics $(n=6)$.

\begin{tabular}{lc}
\hline Characteristics & $\mathrm{N}$ \\
\hline Age (years) & \\
Median & 53 \\
Range & $41-61$ \\
ECOG PS & \\
0 & 5 \\
1 & 1 \\
Clinical tumor stage & \\
T2 & 1 \\
T3 & 0 \\
T4 & 5
\end{tabular}

Clinical nodal status

$\mathrm{N} 1$

$\mathrm{N} 2 \quad 3$

N3 2

ER receptor status

Positive 3

Negative $\quad 3$

PR receptor status

Positive 2

Negative $\quad 4$

Menopausal status

Premenopausal $\quad 3$

Postmenopausal 3

Treatment cycles

Median 4

Range 3-6

Reduction rate (\%)

Median

Range

$39.0-69.9$

ECOG, Eastern Cooperative Oncology Group; PS, performance status; ER, estrogen receptor; PR, progesterone receptor.

Currently, primary systemic chemotherapy with trastuzumab is a standard treatment approach for HER2-overexpressing locally advanced breast cancer. Patients with HER2-positive breast cancer, particularly those with lymph node metastasis, usually have a poor prognosis. However, the introduction of trastuzumab, which was developed to specifically target HER2, has significantly improved the prognosis of patients with this type of cancer (7). Patients with locally advanced HER2-positive tumors at presentation should be treated with primary systemic chemotherapy with trastuzumab, followed by multimodal treatment (8). Preoperative systemic therapy may render surgically inoperable tumors operable due to downstaging and has several potential benefits, including increased chance of survival. Therefore, intensive primary systemic chemotherapy with anti-HER2 agents is important.

The preferred regimens for HER2-positive disease include doxorubicin plus cyclophosphamide followed by paclitaxel 
Table II. Adverse events occurring in $\geq 1$ patients.

\begin{tabular}{|c|c|c|c|c|c|c|c|c|}
\hline \multirow[b]{2}{*}{ Adverse events } & \multicolumn{4}{|c|}{$\begin{array}{l}\text { Level } 1(n=3) \\
\text { Grade }\end{array}$} & \multicolumn{4}{|c|}{$\begin{array}{c}\text { Level } 2(\mathrm{n}=3) \\
\text { Grade }\end{array}$} \\
\hline & 1 & 2 & 3 & 4 & 1 & 2 & 3 & 4 \\
\hline \multicolumn{9}{|l|}{ Hematological } \\
\hline Leukopenia & & & 2 & & & & 2 & \\
\hline Neutropenia & 1 & & 1 & 1 & & & 1 & 1 \\
\hline Decreased hemoglobin & & & & 3 & 1 & & 2 & \\
\hline Thrombocytopenia & 1 & & & 2 & 1 & & 2 & \\
\hline AST/ALT elevation & 1 & 1 & & & & & 2 & \\
\hline Increased creatinine & 1 & & & & & & & \\
\hline \multicolumn{8}{|l|}{ Nonhematological } & \\
\hline Fatigue & 1 & 2 & & & & & & \\
\hline Fever & 1 & & & & & & & \\
\hline Nausea & 2 & & & & & & & \\
\hline Vomiting & & & & & 1 & & & \\
\hline Anorexia & 1 & & & & & 1 & & \\
\hline Malaise & 1 & & & & & & & \\
\hline Diarrhea & & 1 & & & & & & \\
\hline Constipation & 1 & & & & 2 & & & \\
\hline Peripheral sensory neuropathy & 3 & & & & 2 & 1 & & \\
\hline Muscle pain & 1 & & & & 1 & & 1 & \\
\hline Arthralgia & 1 & & & & 1 & & 1 & \\
\hline Alopecia & 1 & 1 & & & 1 & 1 & & \\
\hline Rash & & & & & & 1 & & \\
\hline Stomatitis & & 1 & & & 1 & & & \\
\hline Dysgeusia & 1 & & & & & & & \\
\hline
\end{tabular}

AST, aspartate aminotransferase; ALT, alanine aminotransferase.

plus trastuzumab, or TCH. A previous study (2) demonstrated that a non-anthracycline regimen, $\mathrm{TCH}$, demonstrated a similar efficacy as the anthracycline-based regimens and had a lower risk of cardiotoxicity and leukemia. In addition, another study (4) demonstrated that tri-weekly nab-paclitaxel $\left(260 \mathrm{mg} / \mathrm{m}^{2}\right)$ produced significantly higher response rates and had a favorable safety profile compared with standard paclitaxel $\left(175 \mathrm{mg} / \mathrm{m}^{2}\right)$ for MBC patients.

Therefore, the combination of nab-paclitaxel, carboplatin and trastuzumab was found to be highly effective and was associated with less cardiotoxicity. As a result, the MTD could not be determined in this study, since no severe adverse events occurred in the first treatment cycle. However, a grade $3 / 4$ decrease in hemoglobin occurred in 5 of the 6 patients and grade 3/4 thrombocytopenia in 4 of the 6 patients after the second cycle. A similar regimen, the combination of taxane plus carboplatin and trastuzumab in the BCIRG-006 trial was associated with only $6.1 \%$ grade $3 / 4$ thrombocytopenia and $5.8 \%$ grade $3 / 4$ anemia (2). Similarly, grade $3 / 4$ decrease in the hemoglobin level and thrombocytopenia occurred in only $2 \%$ of the patients in a previous study by using a combination therapy with docetaxel, carboplatin and trastuzumab (9). In both studies, docetaxel was administered at $75 \mathrm{mg} / \mathrm{m}^{2}$ and carboplatin at 6 AUC. In the CA 024 study (5) comparing docetaxel and nab-paclitaxel as first-line chemotherapy for MBC patients, severe hematological events were significantly frequent in patients who received docetaxel $100 \mathrm{mg} / \mathrm{m}^{2}$ tri-weekly compared with those who received nab-paclitaxel $300 \mathrm{mg} / \mathrm{m}^{2}$ tri-weekly.

The phase II trial that evaluated the efficacy and safety of weekly nab-paclitaxel with carboplatin and trastuzumab (10) reported $6 \%$ grade $3 / 4$ anemia and $3 \%$ grade 3 thrombocytopenia. In that study, nab-paclitaxel was administered at $100 \mathrm{mg} / \mathrm{m}^{2}$ and carboplatin at 2 AUC on days 1,8 and 15 of a 28-day cycle. Therefore, the dose intensity in that study was lower compared with the present study. As no severe anemia or thrombocytopenia were observed in other previous studies evaluating monotherapy with nab-paclitaxel $(4,5)$, the concurrent administration of nab-paclitaxel and carboplatin may induce severe anemia or thrombocytopenia.

However, considering the high efficacy and lower risk of cardiotoxicity and secondary carcinogenesis with the combination therapy with taxane, platinum and trastuzumab, further evaluation of another regimen including weekly administration or a more accurate dose setting should be conducted. 
In conclusion, although no DLTs were observed in the first cycle of this combination treatment, there were difficulties with continuity due to hematological toxicity, particularly anemia.

\section{Acknowledgements}

The authors would like to express their gratitude to the women who participated in this trial and their family members, in addition to the investigators and all the staff members at the study sites for their contributions to the study. Medical writing service was provided by Cambridge English Correction Service. Kenji Tezuka reports a grant from Taiho Pharmaceutical Co., Ltd.; Tsutomu Takashima reports personal fees from Taiho Pharmaceutical Co., Ltd., Chugai Pharmaceutical Co., Ltd. and Eisai Co., Ltd.; Hidemi Kawajiri reports personal fees from Chugai Pharmaceutical Co., Ltd. and Eisai Co., Ltd.; Seika Tei reports personal fees from Taiho Pharmaceutical Co., Ltd., Chugai Pharmaceutical Co., Ltd. and Eisai Co., Ltd., outside the submitted work; Shigehiko Nishimura reports personal fees from Daiichi Sankyo Healthcare Co., Ltd., Eisai Co., Ltd., Chugai Pharmaceutical Co., Ltd. and Toshiba Medical Systems Co., Ltd.; Shigehito Yamagata reports personal fees from Taiho Pharmaceutical Co., Ltd., Chugai Pharmaceutical Co., Ltd. and Daiichi Sankyo Co., Ltd.; Takeo Nishimori reports personal fees from Taiho Pharmaceutical Co., Ltd. and Eisai Co., Ltd.; Katsumi Ikeda reports personal fees from Toshiba Medical Systems Co. and Tsumura Co., Ltd.; Naoyoshi Onoda reports grants and personal fees from Bayer, personal fees from Eisai Co., Ltd. and non-financial support from Novartis Pharma K.K.

\section{References}

1. Lee MC and Newman LA: Management of patients with locally advanced breast cancer. Surg Clin North Am 87: 379-398, ix, 2007.
2. Slamon D, Eiermann W, Robert N, Pienkowski T, Martin M, Press M, Mackey J, Glaspy J, Chan A, Pawlicki M, et al: Adjuvant trastuzumab in HER2-positive breast cancer. N Engl J Med 365: 1273-1283, 2011

3. Robert N, Leyland-Jones B, Asmar L, Belt R, Ilegbodu D, Loesch D, Raju R, Valentine E, Sayre R, Cobleigh M, et al: Randomized phase iii study of trastuzumab, paclitaxel, and carboplaine compared with trastuzumab and paclitaxel in women with her-2-overexpressing metastatic breast cancer. J Clin Oncol 24: 2786-2792, 2006.

4. Gradishar WJ, Tjulandin S, Davidson N, Shaw H, Desai N, Bhar P, Hawkins M and O'Shaughnessy J: Phase III trial of nanoparticle albumin-bound paclitaxel compared with polyethylated castor oil-based paclitaxel in women with breast cancer. J Clin Oncol 23: 7794-7803, 2005.

5. Gradishar WJ, Krasnojon D, Cheporov S, Makhson AN, Manikhas GM, Clawson A and Bhar P: Significantly longer progression-free survival with nab-paclitaxel compared with docetaxel as first-line therapy for metastatic breast cancer. J Clin Oncol 27: 3611-3619, 2009.

6. Cancer Therapy Evaluation Program (CTEP): Common Terminology Criteria for AdverseEvents (CTCAE) version 4.0., 2015. http://ctep.cancer.gov/protocolDevelopment/electronic applications/ctc.htm\#ctc_40. Accessed October 8, 2015.

7. Dawood S, Broglio K, Buzdar AU, Hortobagyi GN and Giordano SH: Prognosis of women with metastatic breast cancer by HER 2 status and trastuzumab treatment: An institutionalbased review. J Clin Oncol 28: 92-98, 2010.

8. National Comprehensive Cancer Network Clinical Guidelines in Oncology (NCCN Guidelines) Breast Cancer version 2.2016, 2016. http://www.ncen.org/professionals/physician_gls/pdf/ breast.pdf Accessed May 13, 2016.

9. Coudert BP, Largillier R, Arnould L, Chollet P, Campone M, Coeffic D, Priou F, Gligorov J, Martin X, Trillet-Lenoir V, et al: Multicenter phase ii trial of neoadjuvant therapy with trastuzumab, docetaxel, and carboplatin for human epidermal growth factor receptor-2-overexpressing satge ii or iii breat cancer: Result of the GETN (A)-1 trial. J Clin Oncol 25: 2678-2684, 2007.

10. Conlin AK, Seidman AD, Bach A, Lake D, Dickler M, D'Andrea G, Traina T, Danso M, Brufsky AM, Saleh M, et al: Phase II trial of weekly nanoparticle albumin-bound paclitaxel with carboplatin and trastuzumab as first-line therapy for women with her2-overexpressing metastatic breast cancer. Clin Breast Cancer 10: 281-287, 2010. 